International Journal of Pure and Applied Mathematics

Volume $91 \quad$ No. 2 2014, 179-190

ISSN: 1311-8080 (printed version); ISSN: 1314-3395 (on-line version)

url: http://www.ijpam.eu

doi: http://dx.doi.org/10.12732/ijpam.v91i2.4

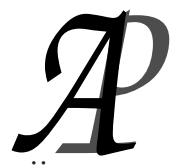

ijpam.eu

\title{
RANDOM FIXED POINT RESULTS FOR SUZUKI TYPE RANDOM OPERATORS AND APPLICATIONS
}

\author{
Renu Chugh ${ }^{1}$, Satish Narwal ${ }^{2}$, Madhu Aggarwal ${ }^{3}$ \\ ${ }^{1}$ Department of Mathematics \\ Maharshi Dayanand University \\ Rohtak, 124 001, Haryana, INDIA \\ ${ }^{2}$ Department of Mathematics \\ Sat Jinda Kalyana College \\ Kalanaur, Rohtak, 124 113, Haryana, INDIA \\ ${ }^{3}$ Department of Mathematics, Vaish College \\ Rohtak, 124 001, Haryana, INDIA
}

\begin{abstract}
In aim of this paper is to prove the random version of Suzuki fixed point theorem in a separable metric space. Our main result generalizes the results of Bharuchareid [1] and Suzuki [22]. Moreover, we show that these maps satisfy property P. Application to certain class of random functional equations arising in dynamical programming is also obtained.
\end{abstract}

AMS Subject Classification: 41A50, 41A65, 47H10, 60H25

Key Words: metric space, random fixed point, random operator, measurable mappings, property $\mathrm{P}$, random functional equation

\section{Introduction}

The research in random fixed point theory has received much attention for

Received: August 26, 2013

(C) 2014 Academic Publications, Ltd.

$\S$ Correspondence author url: www.acadpubl.eu 
the last two decades, especially after the survey article of Bharucha-Reid [1]. The survey article by Bharucha-Reid [1] in 1976 attracted the attention of several mathematicians and gave wings to this theory. Bharucha-Reid proved the stochastic version of the well-known Banach's and Schauder's fixed point theorem and hence random fixed point theory and applications have been developed rapidly in recent years, see e.g., Bharucha-Reid [1, 2, 3], Itoh [20], Papageorgiou [17], Sehgal and Singh [24], Sehgal and Waters [23], and Lin [21]. Random fixed point theorems of Bharucha-Reid [1] are useful for proving the existence results for random solutions of nonlinear random equations in separable Banach spaces. On the other hand, the study of random fixed points and random approximations have gained tremendous importance after the publication of papers by Beg [8, 9], Beg and Shahzad [10, 11, 12, 13, 14, 15], and Xu [6, 7].

The classical Banach fixed point theory states that "every contraction on a complete metric space admits a unique fixed point". Many fixed point theorems have been proved by various authors as generalizations of this result. In 2008, Suzuki [22] introduced a new type of mapping and obtained the following interesting and simple generalization of Banach contraction principle:

Theorem 1.1 ([22]). Define a non-increasing function $\theta$ from $[0,1)$ onto $\left(\frac{1}{2}, 1\right]$ by

$$
\theta(r)= \begin{cases}1 & \text { if } 0 \leq r \leq \frac{1}{2}(\sqrt{5}-1) \\ \frac{1-r}{r^{2}} & \text { if } \frac{1}{2}(\sqrt{5}-1) \leq r \leq \frac{1}{\sqrt{2}} \\ \frac{1}{1+r} & \text { if } \frac{1}{\sqrt{2}} \leq r<1 .\end{cases}
$$

Let $(X, d)$ be a complete metric space and let $T$ be a mappings from $X$ into itself. Assume that there exists $r \in[0,1)$ such that for every $x, y \in X$.

$$
\theta(r) d(x, T x) \leq d(x, y) \text { implies } d(T x, T y) \leq r d(x, y) .
$$

Then there exists a unique fixed point $z$ of $T$. Moreover, $\lim _{n} T^{n}(x)=z$ for all $x \in X$.

Subsequently, Theorem 1.1 was generalized and extended in various ways (see $[4,17,19])$.

In this paper, we prove random analogue of Suzuki fixed point theorem. We also show that these maps satisfy property P. At the end, we obtain application regarding the existence and uniqueness of solutions of certain class of random functional equations arising in dynamical programming.

We now give some basic definitions and notations which will be required in the sequel. 
Let $(X, d)$ be a separable metric space and $(\Omega, \Sigma)$ be a measurable space with $\Sigma$ being a sigma-algebra of subsets of $\Omega$.

Definition 1.1. A mapping $\xi: \Omega \rightarrow X$ is measurable if $\xi^{-1}(U) \in \Sigma$ for each open subset $U$ of $X$.

Definition 1.2. A mapping $T: \Omega \times X \rightarrow X$ is called a random operator if for each $x \in X, T(\cdot, x)$ is measurable.

We denote the $n$th iterate $T(\omega, T(\omega, T(\omega, \ldots, T(\omega, x))))$ of $T$ by $T^{n}(\omega, x)$. The letter $I$ denotes the random mapping $I: \Omega \times X \rightarrow X$ defined by $I(\omega, x)=x$ and $T^{0}=I$.

Definition 1.3. A mapping $T: \Omega \times X \rightarrow X$ is called continuous if for each $\omega \in \Omega$ the mapping $T(\omega, \cdot): X \rightarrow X$ is continuous.

Definition 1.4. A measurable mapping $g: \Omega \rightarrow F$ is the random fixed point of the random operator $T: \Omega \times F \rightarrow X$ if and only if $T(\omega, g(\omega))=g(\omega)$, for each $\omega \in \Omega$. We denote the set of random fixed points of $T$ by $R F(T)$.

Example 1.5. Let $\Omega=[0,1]$ and $\Sigma$ be the sigma algebra of Lebesgue's measurable subsets of $\Omega$. Take $X=R$ with $d(x, y)=|x-y|$, for $x, y \in R$. Define random operator $T$ from $\Omega \times X$ to $X$ as, $T(\omega, x)=\omega-x$. Then a measurable mapping $\xi: \Omega \rightarrow X$ defined as $\xi(\omega)=\frac{\omega}{2}$, for every $\omega \in \Omega$, serve as a unique random fixed point of $T$.

Definition 1.6. Let $F$ be a nonempty subset of a separable metric space $X$. The random operator $T: \Omega \times F \rightarrow F$ is said to be $k(\omega)$-contraction random operator if for any $x, y \in F$ and $\omega \in \Omega$, we have

$$
d(T(\omega, x), T(\omega, y)) \leq k(\omega) d(x, y),
$$

where $k: \Omega \rightarrow[0,1)$ is a measurable map.

Definition 1.7. Let $T: \Omega \times X \rightarrow X$ be a random operator with random fixed point set $R F(T) \neq \phi$. Then $T$ is said to have property $P$ if $R F\left(T^{n}\right)=$ $R F(T)$, for each $n \in N$. Equivalently, a mapping has property $\mathrm{P}$ if every periodic point is a fixed point.

An interesting fact about maps satisfying property $\mathrm{P}$ is that none of these maps have any non-trivial periodic points. Some papers dealing with property $\mathrm{P}$ are $[5,18]$. 


\section{Main Results}

Theorem 2.1. Let $X$ be a separable complete metric space and $(\Omega, \Sigma)$ be a measurable space. Let $T: \Omega \times X \rightarrow X$ be a mapping. Define a non-increasing function $\theta$ from $[0,1)$ onto $\left(\frac{1}{2}, 1\right]$ by

$$
\theta(r(\omega))= \begin{cases}1 & \text { if } 0 \leq r(\omega) \leq \frac{1}{2}(\sqrt{5}-1) \\ \frac{1-r(\omega)}{r(\omega)^{2}} & \text { if } \frac{1}{2}(\sqrt{5}-1) \leq r(\omega) \leq \frac{1}{\sqrt{2}} \\ \frac{1}{1+r(\omega)} & \text { if } \frac{1}{\sqrt{2}} \leq r(\omega)<1 .\end{cases}
$$

Assume that for all $\xi, \eta \in X$ and $\omega \in \Omega$,

$$
\theta(r) d(\xi(\omega), T(\omega, \xi(\omega))) \leq d(\xi(\omega), \eta(\omega))
$$

implies that

$$
d(T(\omega, \xi(\omega)), T(\omega, \eta(\omega))) \leq r(\omega) d(\xi(\omega), \eta(\omega))
$$

where $r: \Omega \rightarrow[0,1)$ is a measurable map. Then $T$ has a unique random fixed point.

Proof. Since $\theta(r(\omega)) \leq 1$, so

$$
\theta(r(\omega)) d(\xi(\omega), T(w, \xi(\omega)))<d(\xi(\omega), T(\omega, \xi(\omega)))
$$

holds for every $\xi \in X$ and $\omega \in \Omega$. Thus by (1), we have

$$
d\left(T(\omega, \xi(\omega)), T^{2}(\omega, \xi(\omega))\right) \leq r(\omega) d(\xi(\omega), T(\omega, \xi(\omega))) .
$$

Let $\xi_{0}: \Omega \rightarrow X$ be any fixed measurable mapping. Define the sequence of measurable mappings $\xi_{n}: \Omega \rightarrow X$ as $\xi_{n}(\omega)=T\left(\omega, \xi_{n-1}(\omega)\right)=T^{n}\left(\omega, \xi_{0}(\omega)\right)$ where $n \in N$.

Now, for any $\omega \in \Omega$, we obtain from (2) that

$$
d\left(\xi_{n}(\omega), \xi_{n+1}(\omega)\right) \leq r(\omega)^{n} d\left(\xi(\omega), T\left(\omega, \xi_{n}(\omega)\right)\right)
$$

and hence $\sum_{n=1}^{\infty} d\left(\xi_{n}(\omega), \xi_{n+1}(\omega)\right)<\infty$. Therefore $\left\{\xi_{n}(\omega)\right\}$ is a Cauchy sequence in $X$ for every $\omega \in \Omega$. Since $X$ is a complete metric space, so $\xi_{n}(\omega) \rightarrow u(\omega)$ for every $\omega \in \Omega$, where $u: \Omega \rightarrow X$ being the limit of the sequence of measurable mappings is measurable. 
Next, we show that

$$
d(T(\omega, \xi(\omega)), u(\omega)) \leq r(\omega) d(\xi(\omega), u(\omega)) \text { for all } \xi \in X-\{u\} .
$$

For $\xi \in X-\{u\}$, there exists a $p \in N$ such that

$$
d\left(\xi_{n}(\omega), u(\omega)\right) \leq \frac{d(\xi(\omega), u(\omega))}{3} \text { for all } n \in N \text { with } n \geq p .
$$

Then we have

$$
\begin{aligned}
\theta(r(\omega)) d\left(\xi_{n}(\omega), T\left(\omega, \xi_{n}(\omega)\right)\right) & \leq d\left(\xi_{n}(\omega), T\left(\omega, \xi_{n}(\omega)\right)\right) \\
& =d\left(\xi_{n}(\omega), \xi_{n+1}(\omega)\right) \\
& \leq d\left(\xi_{n}(\omega), u(\omega)\right)+d\left(\xi_{n+1}(\omega), u(\omega)\right) \\
& \leq \frac{2}{3} d(\xi(\omega), u(\omega)) \\
& =d(\xi(\omega), u(\omega))-\frac{d(\xi(\omega), u(\omega))}{3} \\
& \leq d(\xi(\omega), u(\omega))-d\left(\xi_{n}(\omega), u(\omega)\right) \\
& \leq d\left(\xi_{n}(\omega), \xi(\omega)\right) .
\end{aligned}
$$

Thus using (1), we get

$$
d\left(\xi_{n+1}(\omega), T(\omega, \xi(\omega))\right) \leq r(\omega) d\left(\xi_{n}(\omega), \xi(\omega)\right) \text { for } n \geq p .
$$

Letting $n \rightarrow \infty$, we get

$$
d(T(\omega, \xi(\omega)), u(\omega)) \leq r(\omega) d(\xi(\omega), u(\omega)) .
$$

Thus (4) holds for all $\xi \in X-\{u\}$.

Now, we prove that $u(\omega)$ is a fixed point of $T$. We have two cases:

Case (i): When $0 \leq r(\omega)<2^{-\frac{1}{2}}$, then $\theta(r(\omega)) \leq(1-r(\omega)) r(\omega)^{-2}$. Suppose on the contrary that $T(\omega, u(\omega)) \neq u(\omega)$. We first prove that

$$
d\left(T^{n}(\omega, u(\omega), T(\omega, u(\omega)))\right) \leq r(\omega)^{n-1} d(u(\omega), T(\omega, u(\omega)))
$$

and

$$
T^{n}(\omega, u(\omega)) \neq u(\omega) \text { for all } n \in N .
$$

For $n=1$, (5) holds. For $n=2$, (5) follows from (2).

Now, we assume that (5) holds for some $n \in N$ with $n \geq 2$. Since

$$
d(u(\omega), T(\omega, u(\omega)))
$$




$$
\begin{aligned}
& \leq d\left(u(\omega), T^{n}(\omega, u(\omega))\right)+d\left(T^{n}(\omega, u(\omega)), T(\omega, u(\omega))\right) \\
& \leq d\left(u(\omega), T^{n}(\omega, u(\omega))\right)+r(\omega)^{n-1} d(u(\omega), T(\omega, u(\omega))) \\
& \leq d\left(u(\omega), T^{n}(\omega, u(\omega))\right)+r(\omega) d(u(\omega), T(\omega, u(\omega))) .
\end{aligned}
$$

Thus, we have

$$
(1-r(\omega)) d(u(\omega), T(\omega, u(\omega))) \leq d\left(u(\omega), T^{n}(\omega, u(\omega))\right)
$$

Hence

$$
\begin{aligned}
& \theta(r(\omega)) d\left(T^{n}\left(\omega, u(\omega), T^{n+1}(\omega, u(\omega))\right)\right) \\
& \quad \leq\left((1-r(\omega)) r(\omega)^{-2}\right) d\left(T^{n}(\omega, u(\omega)), T^{n+1}(\omega, u(\omega))\right) \\
& \quad \leq\left((1-r(\omega)) r(\omega)^{-2}\right) d\left(T^{n}(\omega, u(\omega)), T^{n+1}(\omega, u(\omega))\right) \\
& \quad \leq(1-r(\omega)) d(u(\omega), T(\omega, u(\omega))) \\
& \quad \leq d(u(\omega), T(\omega, u(\omega))) \\
& \quad=d\left(T^{n}(\omega, u(\omega)), u(\omega)\right) .
\end{aligned}
$$

Using (1) and (4), we have

$$
\begin{aligned}
d\left(T^{n+1}(\omega, u(\omega), T(\omega, u(\omega)))\right) & \leq r(\omega) d\left(T^{n}(\omega, u(\omega)), u(\omega)\right) \\
& \leq r(\omega)^{n} d(T(\omega, u(\omega)), u(\omega)) .
\end{aligned}
$$

Thus by induction, (5) holds for everyn $\in N$.

By $(5), \lim _{n \rightarrow \infty} T^{n}(\omega, u(\omega))=T(\omega, u(\omega))$.

On the other hand from (5), we have $T^{n}(\omega, u(\omega)) \neq u(\omega)$, so applying (4), we get

$$
d\left(T^{n}(\omega, u(\omega)), u(\omega)\right) \leq r(\omega)^{n-1} d(T(\omega, u(\omega)), u(\omega))
$$

Letting $n \rightarrow \infty$, we get $\lim _{n \rightarrow \infty} T^{n}(\omega, u(\omega))=u(\omega)$ and hence $T(\omega, u(\omega))=u(\omega)$ which is a contradiction. Therefore, we have $T(\omega, u(\omega))=u(\omega)$.

Case (ii): When $2^{-\frac{1}{2}} \leq r(\omega)<1$, we assume that for $\xi, \eta \in X$, either

$$
\theta(r(\omega)) d(\xi(\omega), T(\omega, \xi(\omega))) \leq d(\xi(\omega), \eta(\omega))
$$

or

$$
\theta(r(\omega)) d\left(T(\omega, \xi(\omega)), T^{2}(\omega, \xi(\omega))\right) \leq d(T(\omega, \xi(\omega)), \eta(\omega)) \text { holds. }
$$

Because if

$$
\theta(r(\omega)) d(\xi(\omega), T(\omega, \xi(\omega)))>d(\xi(\omega), \eta(\omega))
$$


and

$$
d\left(T(\omega, \xi(\omega)), T^{2}(\omega, \xi(\omega))\right)>d(T(\omega, \xi(\omega)), \eta(\omega)),
$$

then we have

$$
\begin{aligned}
& d(\xi(\omega), T(\omega, \xi(\omega))) \\
& \quad \leq d(\xi(\omega), \eta(\omega))+d(T(\omega, \xi(\omega)), \eta(\omega)) \\
& \quad<\theta(r(\omega))\left(d(\xi(\omega), T(\omega, \xi(\omega)))+d\left(T(\omega, \xi(\omega)), T^{2}(\omega, \xi(\omega))\right)\right) \\
& \quad \leq \theta(r(\omega))(d(\xi(\omega), T(\omega, \xi(\omega)))+r(\omega) d(\xi(\omega), T(\omega, \xi(\omega)))) \\
& \quad=d(\xi(\omega), T(\omega, \xi(\omega))),
\end{aligned}
$$

which is a contradiction.

Hence either

$$
\theta(r(\omega)) d\left(\xi_{2 n}(\omega), \xi_{2 n+1}(\omega)\right) \leq d\left(\xi_{2 n}(\omega), u(\omega)\right)
$$

or

$$
\theta(r(\omega)) d\left(\xi_{2 n+1}(\omega), \xi_{2 n+2}(\omega)\right) \leq d\left(\xi_{2 n+1}(\omega), u(\omega)\right)
$$

holds for every $n \in N$. Using (1), we have either

$$
d\left(\xi_{2 n+1}(\omega)+T(\omega, u(\omega))\right) \leq r(\omega) d\left(\xi_{2 n}(\omega), T(\omega, u(\omega))\right)
$$

or

$$
d\left(\xi_{2 n+2}(\omega), T(\omega, u(\omega))\right) \leq r(\omega) d\left(\xi_{2 n+1}(\omega), u(\omega)\right)
$$

holds for every $n \in N$.

Since $\left\{\xi_{n}(\omega)\right\}$ converges to $u(\omega)$, the above inequalities imply that there is a subsequence of $\left\{\xi_{n}(\omega)\right\}$ which converges to $T(\omega, u(\omega))$. Thus $T(\omega, u(\omega))=u(\omega)$ therefore, we obtain $T(\omega, u(\omega))=u(\omega)$ in both cases. From (4), we obtain that the fixed point of $T$ is unique.

The following random contraction mapping Theorem of of $A$. T. BharuchaReid [1]. follows directly from Theorem 2.1.

Corollary $2.2([1])$. Let $X$ be a separable complete metric space and $(\Omega, \Sigma)$ be a measurable space. Let $T: \Omega \times X \rightarrow X$ be a mapping. Assume that $T$ is a $k(\omega)$-contraction random operator for all $\xi, \eta \in X$ and $\omega \in \Omega$, where $k: \Omega \rightarrow[0,1)$ is a measurable map. Then $T$ has a unique random fixed point.

Now, we shall show that maps satisfying (1) possess property $\mathrm{P}$.

Theorem 2.3. Under the conditions of Theorem 2.1, $T$ has property $P$. 
Proof. From Theorem 2.1, $T$ has a random fixed point. Therefore $R F\left(T^{n}\right) \neq$ $\phi$ for each positive integer $n \geq 1$. Fix a positive integer $n>1$ and let $u(\omega) \in R F\left(T^{n}\right)$, we claim that $u(\omega) \in R F(T)$. Suppose that $T(\omega, u(\omega)) \neq u(\omega)$. Then

$$
\begin{gathered}
\theta(r(\omega)) d\left(T^{n-1}(\omega, u(\omega)), T^{n}(\omega, u(\omega))\right) \\
\quad \leq d\left(T^{n-1}(\omega, u(\omega)), T^{n}(\omega, u(\omega))\right) .
\end{gathered}
$$

Using (1), we have

$$
\begin{aligned}
& d\left(T^{n}(\omega, u(\omega)), T^{n+1}(\omega, u(\omega))\right) \\
& \quad \leq r(\omega) d\left(T^{n-1}(\omega, u(\omega)), T^{n}(\omega, u(\omega))\right) .
\end{aligned}
$$

Continuing like this, we have

$$
d\left(T^{n}(\omega, u(\omega)), T^{n+1}(\omega, u(\omega))\right) \leq r(\omega)^{n} d(u(\omega), T(\omega, u(\omega))) .
$$

that is,

$$
d(u(\omega), T(\omega, u(\omega))) \leq r(\omega)^{n} d(u(\omega), T(\omega, u(\omega)))
$$

which is possible only if $d(u(\omega), T(\omega, u(\omega)))=0$, that is, $T(\omega, u(\omega))=u(\omega)$, which is a contradiction, hence $u(\omega) \in R F(T)$. Therefore $T$ has a property $\mathrm{P}$.

\section{Application}

In this section we prove existence and uniqueness of solution for a class of random functional equations using Theorem 2.1.

Throughout this section, we assume that $U$ and $V$ are Banach spaces, $W \subset$ $U, D \subset V$ and $R$ is the field of real numbers. Let $X=B(W)$ denote the set of all the bounded real valued functions on $W$ with the metric defined as $d(h(\omega), k(\omega))=\sup _{w \in \Omega}|h(\omega)-k(\omega)|$ where $h, k: \Omega \rightarrow X$ are measurable mappings. Then $(X, d)$ becomes a complete metric space. Let $(\Omega, \Sigma, p)$ be a given probability space.

We will study the existence and uniqueness of a solution of the following functional equation

$$
p(x(\omega))=\sup _{y}[g(x(\omega), y(\omega), \omega)+G(x(\omega), y(\omega), p(\tau(x(\omega), y(\omega))))]
$$

where $x: \Omega \rightarrow W$ and $y: \Omega \rightarrow D$ represent the random state and decision vectors respectively, $\tau: W \times D \rightarrow W$ represents the transformation of the 
process and $p(x(\omega))$ represents the optimal return function with initial state. Here $g: W \times D \times \Omega \rightarrow R, G: W \times D \times R \rightarrow R$ are bounded functions.

Let a function $\theta$ be defined as in Theorem 2.1 and let the mapping $T$ : $X \times \Omega \rightarrow X$ be defined as

$$
T(h(\omega), \omega)=\sup _{y \in D}[g(x(\omega), y(\omega), \omega)+G(x(\omega), y(\omega), h(\tau(x(\omega), y(\omega))))]
$$

Theorem 3.1. Suppose that for every $(x, y) \in W \times D, h, k \in X$ and $\omega \in \Omega$, the inequality

$$
\theta(r(\omega)) d(h(\omega), T(h(\omega), \omega)) \leq d(h(\omega), k(\omega))
$$

implies that

$$
d(T(h(\omega), \omega), T(k(\omega), \omega)) \leq r(\omega) d(h(\omega), k(\omega)),
$$

where $r: \Omega \rightarrow[0,1)$ is a measurable map. Then the functional equation (6) has a unique bounded solution in $X$.

Proof. Let $\lambda$ be an arbitrary positive real number and $h_{1}, h_{2}: \Omega \rightarrow X$. For $x(\omega) \in W$, we choose $y_{1}(\omega), y_{2}(\omega) \in D$ and $\omega \in \Omega$ so that

$$
\begin{aligned}
& T\left(h_{1}(\omega), \omega\right)<g\left(x(\omega), y_{1}(\omega), \omega\right)+G\left(x(\omega), y_{1}(\omega), h_{1}\left(\tau_{1}\right)\right)+\lambda \\
& T\left(h_{2}(\omega), \omega\right)<g\left(x(\omega), y_{2}(\omega), \omega\right)+G\left(x(\omega), y_{2}(\omega), h_{2}\left(\tau_{2}\right)\right)+\lambda
\end{aligned}
$$

Where $\tau_{1}=\tau\left(x(\omega), y_{1}(\omega)\right)$ and $\tau_{2}=\tau\left(x(\omega), y_{2}(\omega)\right)$.

From the definition of mapping $T$ and equation (7), we have

$$
\begin{aligned}
& T\left(h_{1}(\omega), \omega\right) \geq g\left(x(\omega), y_{2}(\omega), \omega\right)+G\left(x(\omega), y_{2}(\omega), h_{1}\left(\tau_{2}\right)\right) \\
& T\left(h_{2}(\omega), \omega\right) \geq g\left(x(\omega), y_{1}(\omega), \omega\right)+G\left(x(\omega), y_{1}(\omega), h_{2}\left(\tau_{1}\right)\right)
\end{aligned}
$$

If the inequality (8) holds, then from (9) and (12), we get

$$
\begin{aligned}
T & \left(h_{1}(\omega), \omega\right)-T\left(h_{2}(\omega), \omega\right) \\
& <G\left(x(\omega), y_{1}(\omega), h_{1}\left(\tau_{1}\right)\right)-G\left(x(\omega), y_{1}(\omega), h_{2}\left(\tau_{1}\right)\right)+\lambda \\
& \leq\left|G\left(x(\omega), y_{1}(\omega), h_{1}\left(\tau_{1}\right)\right)-G\left(x(\omega), y_{1}(\omega), h_{2}\left(\tau_{1}\right)\right)\right|+\lambda \\
& \leq r(\omega) d\left(h_{1}(\omega), h_{2}(\omega)\right)+\lambda .
\end{aligned}
$$

Similarly from (10) and (11), we obtain

$$
T\left(h_{2}(\omega), \omega\right)-T\left(h_{1}(\omega), \omega\right) \leq r(\omega) d\left(h_{1}(\omega), h_{2}(\omega)\right)+\lambda
$$


Hence from (13) and (14), we have

$$
\left|T\left(h_{1}(\omega), \omega\right)-T\left(h_{2}(\omega), \omega\right)\right| \leq r(\omega) d\left(h_{1}(\omega), h_{2}(\omega)\right)+\lambda
$$

Since the above inequality is true for any $x(\omega) \in W$ and arbitrary $\lambda \geq 0$, thus we have

$$
\theta(r) d\left(h_{1}(\omega), T\left(h_{1}(\omega), \omega\right)\right) \leq d\left(h_{1}(\omega), h_{2}(\omega)\right)
$$

implies that

$$
d\left(T\left(h_{1}(\omega), \omega\right), T\left(h_{2}(\omega), \omega\right)\right) \leq r(\omega) d\left(h_{1}(\omega), h_{2}(\omega)\right) .
$$

Therefore all the conditions of the Theorem 2.1 are satisfied for the mapping $T$ and hence the random functional equation (6) has a unique bounded solution.

\section{References}

[1] A.T. Bharucha-Reid, Fixed point theorems in probabilistic analysis, Bull. Amer. Math. Soc., 82 (1976), 641-657.

[2] A.T. Bharucha-Reid, Random Integral Equations, Academic Press, New York (1972).

[3] B.S. Choudhary, A common unique fixed point theorem for two random operators in Hilbert space, IJMMS, 32, No. 3 (2002), 177-182.

[4] G. Mot, A. Petrusel, Fixed point theory for a new type of contractive multivalued operators, Nonlinear Analysis: Theory, Methods \& Appl., 70, No. 9 (2009), 3371-3377.

[5] G.S. Jeong, B.E. Rhoades, Maps for which $F(T)=F\left(T^{n}\right)$, Fixed Point Theory and Applications, 6 (2004), 71-105.

[6] H.K. Xu, Viscosity approximation methods for nonexpansive mappings, $J$. Math. Anal. Appl., 298 (2004), 279-291.

[7] H.K. Xu, Some random fixed point theorems for condensing and nonexpansive operators, Proc. Amer. Math. Soc., 110, No. 2 (1990), 395-400.

[8] I. Beg, Minimal displacement of random variables under Lipschitz random maps, Topol Methods Nonlinear Anal., 19 (2002), 391-397. 
[9] I. Beg, Approximation of random fixed points in normed spaces, Nonlinear Anal., 51 (2002), 1363-1372.

[10] I. Beg, N. Shahzad, Random fixed point theorems for nonexpansive and contractive type random operators on Banach spaces, J. Appl. Math. Stochastic Anal., 7 (1994), 569-580.

[11] I. Beg, N. Shahzad, Random fixed point theorems on product spaces, J. Appl. Math. Stochastic Anal., 6 (1993), 95-106.

[12] I. Beg, N. Shahzad, Common random fixed points of random multivalued operators on metric spaces, Bulletin of U.M.J., 7 (1995), 493-503.

[13] I. Beg, N. Shahzad, Random fixed points of random multivalued operators on Polish spaces, Nonlinear Anal., 20, No. 7 (1993), 835-847.

[14] I. Beg, N. Shahzad, Random approximations and random fixed point theorems, J. Appl. Math. Stochastic Anal., 7, No. 2 (1994), 145-150.

[15] I. Beg, N. Shahzad, Random fixed points of weakly inward operators in conical shells, J. Appl. Math. Stoch. Anal., 8 (1995), 261-264.

[16] N.S. Papageorgiou, Random fixed point theorems for measurable multifunction in Banach space, Proc. Amer. Math. Soc., 97, No. 3 (1986), 507514.

[17] O. Popescu, Two fixed point theorems for generalized contractions with constants in complete metric, space, Central European Journal of Mathematics, 7, No. 3 (2009), 529-538.

[18] R. Chugh, R. Kamal, M. Aggarwal, Properties P and Q for Suzuki-type fixed point theorems in metric spaces, International Journal of Computer Applications, 50, No. 1 (July 2012), 44-48.

[19] S. Dhompongsa, H. Yingtaweesittikul, Fixed points for multivalued mappings and the metric completeness, Fixed Point Theory and Applications, 2009, Article ID 972395 (2009), 15 pages.

[20] S. Itoh, A random fixed point theorem for a multivalued contraction mapping, Pacific J. Math., 68 (1977), 85-90.

[21] T.C. Lin, Random approximations and random fixed point theorems for non-self maps, Proc. Amer. Math. Soc., 103 (1988), 1129-1135. 
[22] T. Suzuki, A generalized banach contraction principle that characterizes metric completeness, Proceedings of the American mathematical Society, 136, No. 5 (2008), 1861-1869.

[23] V.M. Sehgal, C. Waters, Some random fixed point theorems for condensing operators, Proc. Amer. Math. Soc., 90, No. 3 (1984), 425-429.

[24] V.M. Sehgal, S.P. Singh, On random approximation and a random fixed point theorem for a set valued mappings, Proc. Amer. Math. Soc., 95 (1985), 91-94. 